site de la part des ingénieurs un efforl permanent, il existe un autre genre de phénomènes qui s'imposent d'cux-mêmes a. lattention par leur brulalité. Il s'agil des accidents.

Gharue accident de construction comporte un enseignement. Pourquoi ne pas en tirer parti? L'idée est belle et a élé développée avec lalent et énergic par M. von Emperger, auquel on doit tant d'initialives intéressantes.

Présentée à la Commission du béton armé, dès le débul de ses Iravaux, elle a fait l'objet de rapports au Congrès de Copenhague el de New-York.

L'intervention d'un organisme international est, en effet, indispensable pour réunir des observations en quantité suflisinte pour on tirer des conclusions générales. Il n’est rien de plus difficile, en effet, que d'obtenir des renseignements cancts stur un accident. Les coupables font toul pour cacher la vérité el les viclimes sont souvent incompélentes. Guant aux experts, le secret professionnel ne leur permet fas de tont divulguer. A côté des accidents importants, dont la presse se fait l'écho plus ou moins fidèle, il y a des accillents courants dont on ne parle pas et à tort car ce sont cux qui forment les vrais constructeurs. De quel intérêt serait crpendant une statistique sincère et détaillée de tous les accidents du bélon armé, qu'il s'agisse de causes inévifables (trumblements de terre, inondations, foudre, incendir, explosions, elc.) ou de causes évitables (défaut du coffruge, fablesse des supports, erreurs de calcul, défectuosité Wu béton, destruction du fer, etc.).

Mais il no faut pas s'altendre à des résultats sérieux sans lintervention des pouvoirs publics. Ce sont les administralions qui doivent organiser un contrôle suffisant pour permeltre de dresser une statistique officielle et, par là même, impartiale de toules les circonstances des accidents. A ce propos, il est surprenant de constater que dans certains jays, la police des constructions est réellement inexistante alor's qu'clle est parfaitement organisée pour d'autres objets twhniques, tols que les chaudières à vapeur, les mines, etc. Pourvu qu'ils observent l'alignement, le propriétaire et l'architede sont libres, si la cupidité ou l'ignorance les y poussent, d'ćdilicr un château de cartes. La répression ne les allend que s'il y a dommage à des personnes ou à des propriélés.

Les efforts faits dans cet ordre d'idées ont été couronnés d'un ploin succès dans deux pays qui, d'ailleurs, depuis longtemps, disposent d'une police des constructions. Une circulaire du Ministre des Travaux publics de Prusse en dile du is seplembre I9I a fixé la procédure à suivre pour lous les accidents aux constructions, même ceux n'ayant cntraîné aucun accident de personnes et a adressé unc liste d'cxperls chargés de l'apprécialion compélente des faits. Une ordonnance analogue, mais plus générale, avait. élé promulguée le 8 mai rg r par le Ministère des Travaux publics d'Autriche.

La question est done résolue dans ces deux pays. Pour les autres il n'est pas à conseiller de produire des rapports privés dont les sources d'information ne sont pas sûres el peuvent donner lieu à róclamations. On ne peut donc qu'émeltre le vou de voir les nations latines suivre l'exemple des nations germaniques. Ce n'est certes pas que leur esprit d'indéprendanoe puisse s'y opposer, puisque, comme nous lo disons plus haut, une telle organisation existe déjà parlout dans des industries anciennes et que probablement le développement rapide de colle du bâtiment est seul cause de linexistence d'une organisation semblable pour elle.

Le Congrès de New-York (VJe Congrès) a donc volé la conclusion suivante:
“ Dans l'intérêl de la prévention d'accidents et en vue de " développer la connaissance des propriétés des matériaux " convenables dans ce but, il paraît indiqué que, dans cha"que pays, la statistique des accidents de construclions soil " organisée comme c’est déjà le cas pour les chaudières à "vapeurr.

"Le Congrès exprime le vou que la Commission du "béton armé travaille à l'organisation d'une telle slalis" lique internationale et présente au prochain Congrès les " rapports, groupés par pays, sur les accidenls, avec les "conclusions à en tirer pour la prévention d'accidents."

Fnsuite de ce vote, le Comité directeur de l'Association internationale a inslitué une sons-commission présidée par M. von Emperger pour l'étude de la question des accidents de béton armé.

\section{I.A CORRECTION DES TORRENTS(1)}

\section{$-($ Suite $)-$}

Dès qu'ils seront stabilisés au moyen de travaux appropriés, los terrains crevassés, ébranlés, qui glissaicnt, scront plantés. Au fur el à mesure que se développera Ja végélation ligneuse, le régime du cours d'eạu se régularisera ol les berges des régions inférieures ćlant moins menacées, la construction de barrages y sera plus facile et moins onćrcuse.

L'érosion régressive est assez lente, c'est une usure du lit, ; l'érosion torrentielle est soudaine, brutale, puissante : celloci agit comme une gonge sur le bois, l'autre, commo dis papien de verre. Contre laquelle faut-jl lutcer d'abord ? la réponse ne saurait être doutouse; il faul aller au plus pressé, mais de là à conclure fu'j] faille négliger l'ćrosion régressive, il y a un abîme.

Est-ce à dire encore qu'on ne doive jamais commencer une correction par le bas ? Co serait aussi exagérć que de vouloir l'imposer dans tous les cas. Chaque torrent a ses caraclères spécifiques de climat, de sol, de pente, d'exposition, d'altitude, de dénudation. Ises travaux à cxéculer dojvent être décidés en Lenant compte de lous ces élémonls. Ici encore, il faut redouter les conceptions purement thériques dans la recherche de la solution : c'est l'obscrvation directe des faits qui doit servir de guide et voici les conclusions auxquelles élait arrivé Demonlzey ${ }^{2}$ ).

"Quant à la marche à suivre dans les travaux, l'expérience a démontré :

" I Q Qu'il importe, avant tout, de conriger tous les ravins supérieurs tributaires d'un torrent donné ;

“ $2^{\circ} \mathrm{Quc}$, dans le lit principal, on doit procéder de l'amont vers l'aval, en ce qui concerne les différenles sections.

" $3^{\circ}$ Qu'au contraire, dans les combes, les travaux do l'amont devant s'appuyer sur ceux d'aval, i] y a licu do procéder généralement du bas vers le haut, à l'exception des combes sèches où l'on doit exclusivement reprendre les travaux par le haut;

" $4^{\circ}$ Que dans le cas de glissement sur les versants du torrent, chaque section doit être traitéo comme une combe;

" $5^{\circ}$ Que dans chaque section, les travaux secondaires seront toujours menés de J'aval vers l'amont entre deux barrages consécutifs. " .

(1) Par M. P. Mougrn : Rapport au Congrès interrational forostien. (2) Lextinction des torrents en France par lo reboisement (Paris, Imnrim ric Nalimale 1894, p. 87. 
L.es résultats donnés par celle méthode éclectique n'ont pas été lels qu'il faille y renoncer. Beaucoup de corrections de torrents ont été rćussies, qui onl été commencées par le haul. Mais il ne faut pas, une fois les travaux entrepris, les cntrecouper, comme cela est malheureusement arrivé, par des intervalles d'inaction qui donnent à l'érosion régressive le temps d'intervenir. Ce sont ces intermittences, non moins que l'étriquement des périmètres, qui ont causé des mécomples.

I.es corrections per descensum se terminent d'ordinaire par l'établissement d'un solide ouvrage de base qui sert alors de couronnement à l'édifice au lieu d'en être le point de départ comme dans la méthode per ascensum. Dans les deux cas, cet ouvrage de base est l'obstacle posé par l'hom me à l'aclion de l'ćrosion régressive.

En résumé, c'est au technicien à décider de l'opportunito de commencer les travaux de correction d'un torrent par le haut ou par le bas d'apres l'examen des circonstances locales el non d'après une formule théorique qui, on vient de le voir, n'envisage qu'une des données du problème.

\section{LES CORRECTIONS D'AVALANGIES}

Dans les régions montagneuses, en dehors des érosions et des ravages lorrentiels, il est d'autres phénomènes qui, chaque annéc, font des victimes, arrêtent la circulation et endommagent les propriétés de tous genres : ce sont.les avalanches.

Tombées sur les versants dénudés ou non boisés des monlagnes, les neiges sont sollicitées sans cesse par l'action de la pesanteur et, bien souvent, avant qu'elles aient pu fondre, elles se précipitent, en nappes ou en lourbillons vers la profondeur des vallons.

Lcurs dégâts sont loin d'être négligeables.

Ainsi de 1900 à 1912 , dans les seuls départements de la Savois, elles ont dévasté i 3 ro hectares de forêls, renversant, brisant 2 s 056 mètres cubes de bois ; elles ont délruit ou rndommagé r34 bâtiments divers, enseveli r 28 personnes (dont 27 ont péri) el 203 animaux domestiques de loutes espèces ; elles ont barré 569 fois des cours d'eau et 522 fois des voies de communication de terre ou de fer.

Dans la scule commune de Chamonix, par exemple, les avalanches ont créé dans la zone forestière des couloirs qui n'occupent pas moins de 3oo hectares ainsi frappés d'une stérilité presque totale; elles cmpêchent en hiver la circulalion des trains en amont de cetle importante localité et menacent de ruine de nombreux hameaux.

Ce n'est pas sculement par la destruclion des forêts que les avalanches favorisent le développement et les ravages des torrents; en roulant sur les pentes, elles arrachent des rochers, labourent les portions terreuses nues et elles accumulent dans les thalwegs d'énormes quantilés de matériaux. I.es brusques fontes des neiges ou les "sacs d'eau " de l'été remanient ces dépòts meubles qui augmentent le volume et l'importance des laves torrentiolles. D'après les observations failes en Savoit depuis quelques annces, on a noté que certaines avalanches de fond ou de glacier pouvaient entraîner jusqu'à 8.000 mòtres cubes de blocs, de gravier of de terre ?

De tels apports sont donc loin d'être négligeables!

Souvent aussi les avalanches conslituent les plus séricux obslacle au reboisement.

De méme que pour les torrents, Ics montagnards avaient depuis longtemps cherché a se garantir de leur choc par des Iravaux édifiés à proximité mêne des points à protéger (tournes, épcrons). Ils n'avaicnt pas été sans remarquer qur ces phénomènes ne prenaicnt pas naissance dans les partics boisées : aussi les cantons silués au-dessus des villages et des hameaux menacés par les neiges avajent-ils, de bonne heure, été placés hors des cxploitations pour former des forêts de protection, des bois de ban ou bois bannis.

Nalurellement aussi on en vint à conclure que pour arrêt: ter, prévenir les avalanches, il fallail reconstituer les massifs imprudemment détruils; mais bien vite on s'aperçut que la reforestation n'étail possible qu'à la condition de retenir les neiges sur les pentes jusqu'à ce que les plantations fussent assez forles pour jouer leur rôle.

Ici encore les travaux de correction doivent précéder les travaux forestiers ; parfois même, lorsque lc bassin de formation de l'avalanche se trouve à une altitude supérieure à la limite de la végétation forestière, sont-ils les sculs à entreprendre.

G'est en Suisse que les corrections d'avalanches sont les plus nombreuses et les plus importantes; la France et l'Autriche on offrent aussi divers exemples.

Le principe de tous les ouvrages de correction est de fixér les neiges sur les pentes.

On y arrive :

I $^{\circ}$ En ménageant sur les versants des plates-formes horjzontales plus ou moins longues, larges de I m. 50 au moins, disposées en chicane à des nivcaux différents dans la région d'où partent les neiges : ce sont les banquettes. Elles sont, tantôt entièrement en déblai, tantôt partic en déblai partie on remblai ; clles permeltent de donner de l'assiette aux nappes neigeuses.

$2^{\circ}$ En édifiant sur les pentes des obstacles artificiels munis d'une berme large d'environ $\mathrm{r}$ mètre, à l'amont, dont la hauteur au-dessus du sol est fonction de l'importance des précipitalions neigeuses à l'endroit considéré.

I e choix du procédé se base sur des observations locales: abondance ou rareté de pierres de bonne qualité, présence ou absence de massifs forestiers à proximité, etc. Ces divers moyens peuvent être employés, tantôt séparément, tantốt concurremment. Le type initial peut être modifié suivant les circonstances : à la banquette, on assimile la passerelle à neige faite d'une forle perche placée horizontalement, supportant des rondis ou des branches dont l'autre extrémité repose sur le sol (Schneebrücke).

Lc rateau est une sorte de passerelle où les rondins placés presque perpendiculairement au sol et assez distants retiennent de grandes quanlités de neige.

Des grillages à larges mailles sont aussi die précieux moyens de rétention.

Au simple mur sec, on peut substitucr de fortes levéç de terre, couvertes de gazon, parfois couronnées d'une rángée de pilots, de manière à accroître le relief de l'ouvrage:

Des murs de soutènement élablis à l'amont et à l'aval permetlent, sans remuer un trop grand cube de terre, d'édifier de vérilables remparts.

Il peut arriver que l'avalanche se détache de parties inaccossibles ou d'un cirque trop ćtendu : dans ce cas, au moyen de véritables digues ou épis, on se borne à en diriger lể cours vers un gouffre, un ravin, ò̀ elle ne saurait causer: de dommages.

Parfois aussi on en barre la route au moyen de muraillements assez puissants pour en arrêter la marche.

Mais qu'il s'agisse de la correction d'un torrent ou de celle d'une avalanche, il semble logique de proportionner l'effort au résullat à obtenir. Ce n'est pas un des côtés les moin difficiles de la question. Si l'on peut estimer avec suffisammient de précision la valeur des propriétés à garantir, celle des surfaces à remettre ensuite en production, comment évaluer les interruptions de la circulation? 
S'il s'agit de voies ferrées servant au commerce international, l'arrêt du trafic, les détournements dies voyageurs et des marchandises peuvent entraîner des pertes énormes.

Il faut aussi envisager l'intérêt général : comment calculer le préjudice causé au pays par la suspension des communications au moment d'un conflit ? d'une guerre ? Dans quelle mesure des laves torrentielles, des avalanches peuvent-elles agir sur le régime des rivières et des fleuves ?

Enfin, lorsque la vie humaine est en jeu, et ce cas n'est malheurcusement que trop fréquent, ne devra-t-elle donc compter pour rien ?

On a vu plus haut la nécessité d'exécuter des travaux de correction. Il n'y a pas plus de raison de les repousser que d'interdire à un malade une opération chirurgicale.

Que de tcls ouvrages soient coûleux, nul ne le nie; quo des écoles aient été faites ct que des fautes aient été commises, il n'en pouvait être autrement en un art si réccnt où tout ćail à créer. et surtout dans les cas où, sous la pression du public, de ses représentants, l'Administration a cu à intervenir sans délai !

Mais dès que l'on a reconnu l'utilité de traiter un torrent, il faut, sans luxe, exécuter lous les travaux indispensables. A ne faire les choses qu'à demi, on risque de tout voir disparailre dans un retour offensif du torrent ou au moins d'être obligé de revenir exécuter deis ouvrages complémentaires et de n'avoir en définitive qu'une correction moins homogène et plus dispendieuse que celle qu'il eût fallì prévoir !

Ce que l'on peut demander raisonnablement, c'est que lon n'entreprenne la correction d'un torrent qu'après une enquête approfondic sur la nécessité de l'opération et une ćlude complète des móyens à appliquer pour atteindre le but. Il ne manque heureusement pas de torrents où les corrections ont donné tout ce qu'on en attendait, où devant les résultats acquis, les populations, jadis les adversaires du Service forestier, en sont devenues les plus précieux auxiliaires et où les faits donnent le plus éclatant démenti aux affirmations erronées el au pessimisme tendancieux de ceux qui nient, de parti pris, l'efficacité et jusqu'à l'utilité même de la consolidation et de la restauration des terrains en montagnes.

\section{LES TORRENTS DE LA SAVOIE -( Suite ) -}

Limite acluelle des Neiges persistantes. - La limite des neiges persistantes est la ligne au-dessus de laciuelle la chaleur estivale est incapable de fondre la totalité des neiges de l'hiver. Elle varie avec la latitude, l'exposition, l'altitude et l'ampleur des massifs montagneux, la direction des vents dominants et l'abondance plus ou moins grande de la vapeur d'eau dans l'air.

En Savoie, on ne peut rencontrer cette limite que dans 4 groupes montagneux : $x^{\circ}$ la chaîne du Mont-Blanc ; $2^{\circ}$ les cimes de la Haute-Tarentaise ; $3^{\circ}$ les massifs de la Vanoise et de Polset ; $4^{\circ}$ l'arête frontière de la Haute-Maurienne.

D'une façon générale, ce sont les petits glaciers suspendus qui ont un bassin d'alimentation très réduit qui, par suite, ne sont pas influencés par des apports solides venus de plus haut, qui sont les meilleurs témoins : ce sont ceux sur lesquels l'enneigement et la fusion ont les effets les plus sensibles et les plus rapides.

(i) P. Movgin (Extrait de son ouvrage: Les Tcrrents de la savoie). $r^{\circ}$ Région du Mont-Blanc. - Tous les glaciers, tant de Ja chaine du Mont-Blane que de celle des Aiguilles-Rouges, sont en régression marquée. Au glacier de Tête-Rousse, oi les observations méthodiques sont faites tous les ans depuis rgor, les précipitations neigeuses hivernales ont été conplètement fondues pondant les étés de rgor, rgo3, rgo., rgo5 et rgo6. A la fin de septembre 1902 et rgo7, r9o8, I909, il restait un résidu neigeux des précédents hivers. Il en résulte que le glacier de Tête-Rousse dont l'altitude moyenne est de 3200 mètres se trouve, à très peu près, à la limite des neiges persistantes.

La diminution considérable des glaciers du Dru, de la Pendant, du Nant-Blanc, au nord de la chaine du MontBlanc, de la Floria et des Aiguilles-Rouges, qui arrivent ì 3 ooo mètres an-dessus du niveau de la mer, montrent nettement que la limite des neiges persistantes est cerlainement bien au-dessus de la cole 3 ooo mètres.

Quelque trente ans auparavant, on assignait comme limile des neiges persistantes, dans la vallée de Chamonix, la cole 2750 mètres.

$2^{\circ}$ Haute-Tarentaise. - Sur les versants ensoleillés, Ies glaciers suspendus ont fortement reculé ct lour régrossion se fait sentir même au-dessus de 3 ooo mèlres ; au contrairc, ccrlains glaciers exposés au Nord n'ont guère varié à parlir de 2 goo mètres.

$3^{\circ}$ Massif de la Vanoise et do Polset. - Silués cntre les vallées du Doron-de-Bozel el de l'Arc, les glaciers suspendus de ces massifs ont subi une fusion intense et mème ont presque disparu : ainsi le glacier de la Masse $\left(2806^{\mathrm{m}}\right)$, lo glacier de Vallée-Etroite $\left(29^{85^{\mathrm{m}}}\right.$ ), le glacier de la cime de Carron $\left(3 \times 49^{\mathrm{m}}\right)$. Ici encore la limite d.es neiges persistantes a dépassé 3 roo mètres.

Haute-Maurienne. - Les glaciers du fond de la vallée de l'Arc, en amont de Bessans, sont en général des glaciers de plateau largement étalés, dont les langues terminales, jadis fort développées, se sont retirées juscju'au niveau du socle montagneux qui supporte l'arête frontière, vers l'altitude de 2700 mètres. Mais le recul s'est fait sentir bien plus haut : ainsi on voit maintenant apparaître entre les glaciers des sources de l'Are et du Mulinet une barre rocheuse, à la cole de 3 ooo mètres, autrefois cachée sous une nappe glacée continue.

Les petils glaciers suspendus de toute la chaîne frontière ont subi une fusion énergique. Depuis les glaciers de la Levanna " presque réduits à rien " jusqu'au glacicr de la BellePlinier $\left(3 \circ \mathrm{og}^{\mathrm{m}}\right)$, du grand Vallon $\left(3 \mathrm{~s} 3 \mathrm{~h}^{\mathrm{m}}\right)$ et du Thabor $\left(3205^{\mathrm{m}}\right)$, tous les appareils glaciaires témoignent que l'ablation l'a de beaucoup emporté sur l'alimentation et que la limile des neiges persistanies se tronve reporice plus haul que 3.200 mètres.

Ces oscillations de la limite des neiges persistantes peuvent être sans doutc beaucoup plus considérables et on ignore là où s'arrêtait cette limite au moment de la grande extension glaciaire de 1820 .

Actuellement, la zone supérieure forestière ne dépasse pas 2400 mètres en Savoie ; il serait dlonc très hasardeux " de porter le reboisement beaucoup plus haut que ne peu" vent l'indiquer Jes forêts actuelles ct de ne s'arrêter qu'aux " terrains où les neiges sont susceptibles de demeurer pendant plusicurs années de suite ". Comme il semble qu'on ait traversé une période d'enneigement minimum, il pourrait se faire qu'après être montée de cinq cents mètres audessus de l'altitude de 2700 mètres, la limite des neiges persistantes, par une oscillation inverse, redescendit audessous et anéantit les plantations faites, même si elles éus- 